\title{
Therapeutic drug repositioning using personalized proteomics of liquid biopsies
}

\author{
Gabriel Velez, ${ }^{1,2,3}$ Alexander G. Bassuk, ${ }^{4}$ Diana Colgan, ${ }^{1,2}$ Stephen H. Tsang, ${ }^{5,6}$ \\ and Vinit B. Mahajan ${ }^{1,2,7}$ \\ 'Omics Laboratory, Stanford University, Palo Alto, California, USA. ²Department of Ophthalmology, Byers Eye Institute, \\ Stanford University, Palo Alto, California, USA. ${ }^{3}$ Medical Scientist Training Program, and ${ }^{4}$ Department of Pediatrics, \\ University of lowa, lowa City, lowa, USA. ${ }^{5}$ Barbara and Donald Jonas Laboratory of Stem Cells and Regenerative Medicine \\ and Bernard \& Shirlee Brown Glaucoma Laboratory, Edward S. Harkness Eye Institute, and ${ }^{6}$ Department of Pathology \\ $\&$ Cell Biology, College of Physicians \& Surgeons, Columbia University, New York, New York, USA. PPalo Alto Veterans \\ Administration, Palo Alto, California, USA.
}

BACKGROUND. In patients with limited response to conventional therapeutics, repositioning of already approved drugs can bring new, more effective options. Current drug repositioning methods, however, frequently rely on retrospective computational analyses and genetic testing - time consuming methods that delay application of repositioned drugs. Here, we show how proteomic analysis of liquid biopsies successfully guided treatment of neovascular inflammatory vitreoretinopathy (NIV), an inherited autoinflammatory disease with otherwise poor clinical outcomes.

METHODS. Vitreous biopsies from NIV patients were profiled by an antibody array for expression of 200 cytokine-signaling proteins. Non-NIV controls were compared with NIV samples from various stages of disease progression. Patterns were identified by 1-way ANOVA, hierarchical clustering, and pathway analysis. Subjects treated with repositioned therapies were followed longitudinally.

RESULTS. Proteomic profiles revealed molecular pathways in NIV pathologies and implicated superior and inferior targets for therapy. Anti-VECF injections resolved vitreous hemorrhages without the need for vitrectomy surgery. Methotrexate injections reversed inflammatory cell reactions without the side effects of corticosteroids. Anti-IL-6 therapy prevented recurrent fibrosis and retinal detachment where all prior antiinflammatory interventions had failed. The cytokine array also showed that TNF- $\alpha$ levels were normal and that corticosteroid-sensitive pathways were absent in fibrotic NIV, helping explain prior failure of these conventional therapeutic approaches.

CONCLUSIONS. Personalized proteomics can uncover highly personalized therapies for autoinflammatory disease that can be timed with specific pathologic activities. This precision medicine strategy can also help prevent delivery of ineffective drugs. Importantly, proteomic profiling of liquid biopsies offers an endpoint analysis that can directly guide treatment using available drugs.

funding organizations had no role

in design and conduct of the study; collection, management, analysis,

and interpretation of the data; preparation, review, or approval of the manuscript; and decision to submit the manuscript for publication.

Conflict of interest: The authors have declared that no conflict of interest exists.

Submitted: October 3, 2017 Accepted: November 8, 2017 Published: December 21, 2017

Reference information: JCI Insight. 2017;2(24):e97818. https://doi.org/10.1172/jci. insight. 97818

\section{Introduction}

After spending several billion dollars and a decade or more in development and clinical trial, only approximately $10 \%$ of new drugs show efficacy and reach the market for patient use. This apparent inefficiency, however, masks enormous opportunities to repurpose existing drugs to treat diseases for which they were not originally intended, especially diseases with limited therapeutic options $(1,2)$. Therapies for inflammatory diseases are an attractive choice for drug repositioning because of the complexity of the immune response, the hit-or-miss nature of treatment (which is often by trial and error), and the number of traditional drugs and new biologics for inflammatory diseases.

Determining which drugs to reposition, however, is not trivial. Among a heterogeneous patient population that seems affected by the same autoimmune condition, one cannot be sure if the disease is in fact the same, or at what progressive stage a patient is presenting. Currently, most predictions of patients that would benefit from drug repositioning are drawn from retrospective computational methods, genomic analyses, 
and genome-wide association studies (GWAS) and use large study populations to define disease signatures. In practice, however, a patient's genetic profile usually only denotes risk, which may do little to guide treatment in the near term. We have a patient population that offers an opportunity to overcome these challenges, in a family affected by a little understood autoinflammatory genetic disease of the retina, neovascular inflammatory vitreoretinopathy (NIV; OMIM 193235), for which there is no effective therapy. This family is transmitting a single disease mutation, so using them for drug repositioning studies removed a major barrier - patient heterogeneity. Indeed, with the NIV family, the shared genetic origin and highly characteristic phenotypic stages made an ideal population to test whether proteomics can guide rapid drug repositioning.

NIV is a progressive intraocular inflammatory condition that follows a series of predictable pathological stages before eventually leading to blindness. NIV is a dominant genetic condition caused by hyperactivating mutations in calpain-5 (CAPN5), a regulatory protease believed to modulate the function of its proteolytic targets (3). Although NIV-causing mutations have been identified, it is neither known how this overactive protease might cause uveitis (intraocular inflammation) nor the identity of its proteolytic targets. Indeed, identification of the disease mutation has done little to inform treatment. This rare disease shares clinical features of several, more common eye pathologies, including retinitis pigmentosa, proliferative diabetic retinopathy, and uveitis. For these diseases, as with NIV, the molecular effectors mediating the symptoms are unknown and treatment is frequently inadequate. NIV patients fail conventional immunosuppressive therapy (e.g., TNF- $\alpha$ inhibitors, and implantable steroid-delivery systems) and all affected patients eventually go blind. Several members in these families are young, and urgently need innovative approaches to therapy.

Since the NIV autoimmune uveitis phenotype can be accurately staged in real time (Figure 1A), we predicted that each stage of affected eyes would have its own proteomic signature of inflammation. Since inflammatory diseases, in general, share the pathological features of NIV (i.e., chronic inflammation and fibrosis) and many relevant drugs and biologics have been developed, we suspected drug repositioning could be guided by cytokine signatures of immune diseases, such as leukocyte infiltration and activation, neovascularization, and end-point fibrosis. We hoped that by identifying cytokine signatures in NIV eyes, we could reposition therapeutic options, in real time.

\section{Results}

Personalized proteome of liquid biopsies identifies differentially expressed cytokine-signaling proteins in NIV stages. We worked under the assumption that our patients might benefit from already available therapeutics. To increase therapeutic potency, we focused on finding targets that could be delivered directly by intravitreal injection, because the blood-ocular barriers prevent many drugs from entering the eye. Injection into the vitreous allows high local concentrations of drugs without the side effects of systemic delivery. In addition to small molecules, we considered injectable antibodies that target abnormally upregulated cytokines. To find which cytokine signals should be targeted, liquid biopsies from patient eyes were screened using a proteomic platform that can monitor hundreds of cytokine signals simultaneously - a precision medicine strategy.

Vitreous biopsies were collected from 8 NIV eyes and 4 eyes in 4 patients with noninflammatory disease (i.e., an epiretinal membrane or macular hole). Three of the NIV eyes were in stage II, 4 in stage III, and 1 in IV (Figure 1A and Supplemental Table 1; supplemental material available online with this article; https://doi.org/10.1172/jci.insight.97818DS1). Biopsies were analyzed using a membrane-based antibody array, to identify any abnormally expressed cytokine-signaling proteins, and determine a NIV protein signature. Additional information concerning the performance of the array is included in the online supplement (Supplemental Tables 2-4 and Supplemental Figure 1). Biopsies from 4 control eyes were compared with 8 NIV eyes, using 1-way ANOVA and hierarchical heatmap clustering.

Our analysis of NIV eyes revealed 64 differentially expressed proteins $(P<0.05): 3$ were downregulated and 61 were upregulated (Figure 1B). In hierarchical heatmap clustering, control samples grouped separately from NIV samples, showing that each group shared specific protein expression patterns.

Rational molecular therapy: targeting differentially expressed proteins. Using the cytokine-signaling expression analysis, we assembled a list of proteins that might be targeted by injectable antibodies that are already clinically in use. We first noticed that, in all the NIV eyes, TNF- $\alpha$ levels were normal (Figure 2A). This explained the previous failure to control inflammation in our patients with infliximab (anti-TNF- $\alpha$ ) infusions, a common therapy used for autoinflammatory diseases that do not respond to conventional immunosuppression. This bolstered our confidence in the sensitivity and accuracy of our proteomic approach. 
A Clinical Phenotype

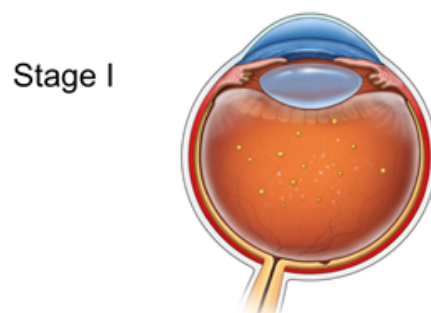

Stage II

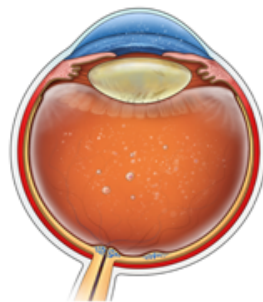

Early cataract

Moderate inflammatory cells

Retinal edema

Early pigmentary retinopathy

B Molecular Phenotype

Mild inflammatory cells Synaptic signaling defect Retinal vasculitis

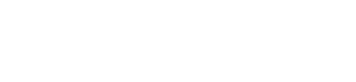

Moderate inflammatory cells Pigmentary retinopathy Neovascularization Early fibrosis Vitreous bands

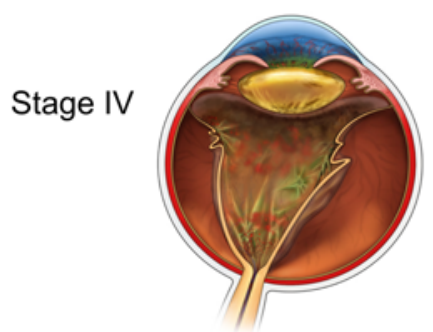

Stage V

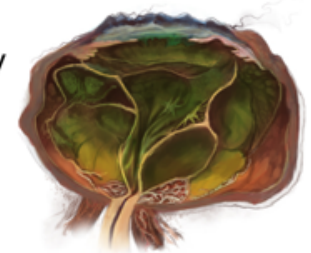

Advanced cataract Moderate inflammatory cells Vitreous hemorrhage Severe fibrosis Retinal detachment Neovascular glaucoma

Phthisis

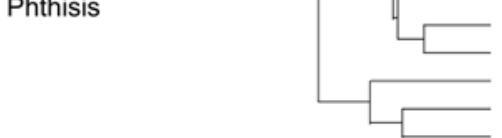

Increasing Disease Severity...............
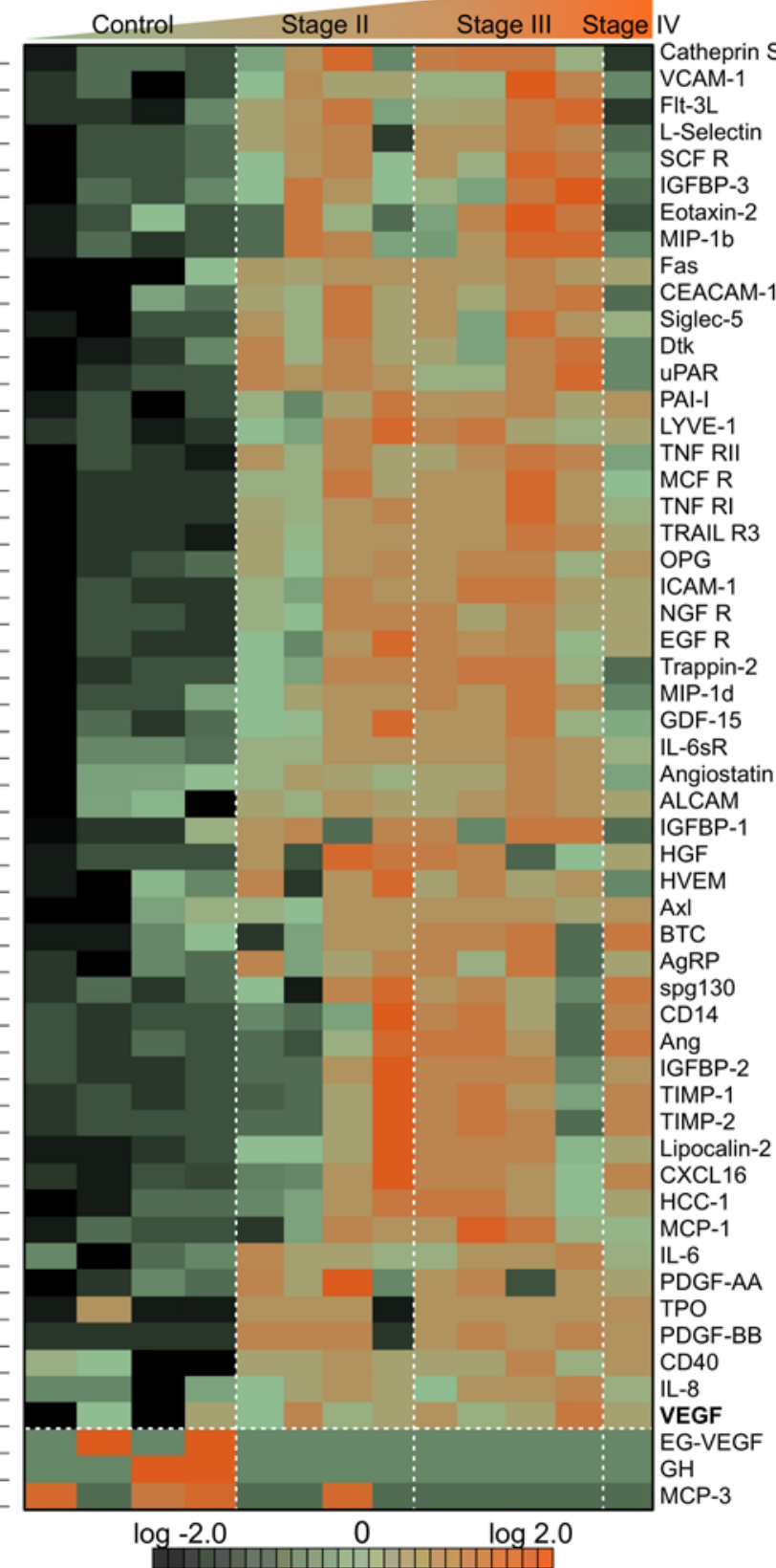

Figure 1. Vitreous proteomics identifies a unique cytokine signature associated with an autoinflammatory uveitis syndrome. (A) Clinical features of neovascular inflammatory vitreoretinopathy (NIV), a blinding uveitis, share the hallmarks of many other autoimmune diseases: infiltration by inflammatory cells, edema, neovascularization, and fibrosis. (B) Cytokine-expression profiles of eyes affected by NIV, an autoinflammatory uveitis (4 technical replicates per sample). Hierarchal clustering shows 64 cytokines expressed differentially $(P<0.05)$ in 8 NIV eyes versus 4 control eyes. Of these cytokines, 3 were downregulated and 61 were upregulated.

VEGF inhibition. Some of our patients were in intermediate NIV stages, when retinal neovascularization develops. The neovascularization causes blinding vitreous hemorrhage. Although the patients did not show evidence of retinal ischemia known to trigger VEGF expression, in the cytokine array, we found consistently and markedly elevated VEGF levels that correlated with disease progression (Figure 2A). The anti-VEGF antibody bevacizumab, originally developed to block tumor blood supply, had been recently repurposed to treat choroidal neovascularization in age-related macular degeneration (AMD) at the time of the study (4). While NIV patients do not develop choroidal neovascularization, 
A

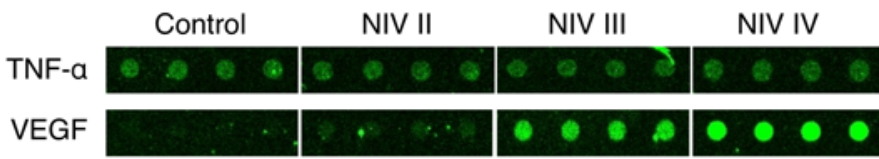
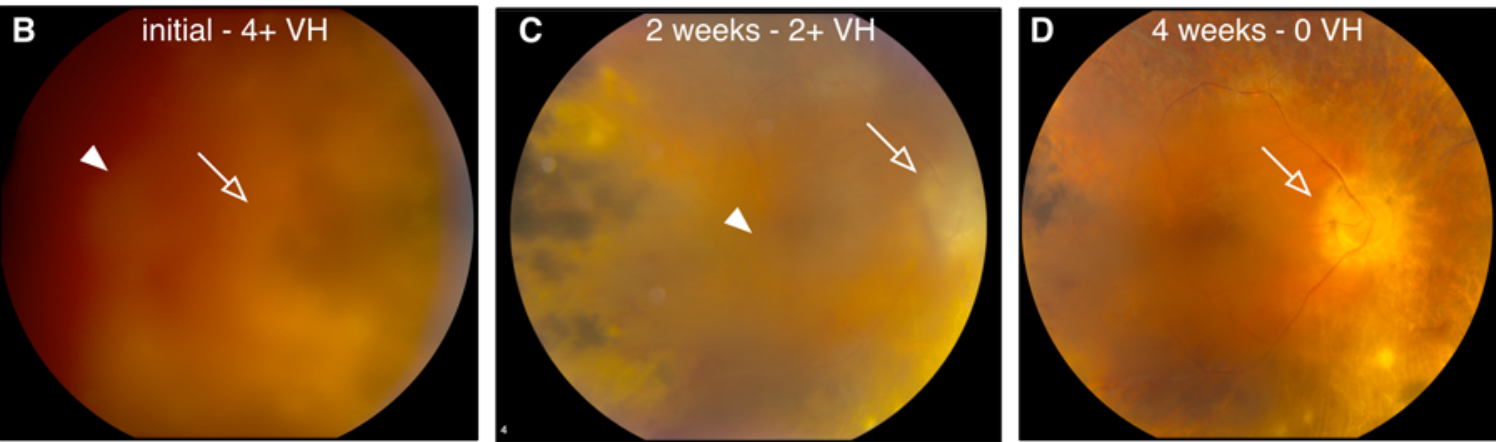

E

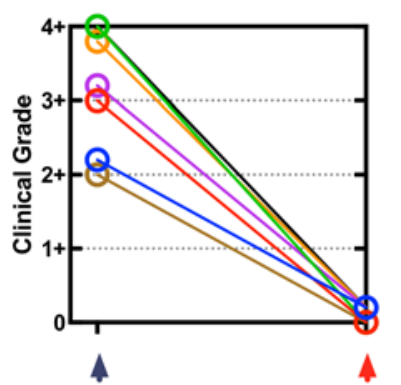

Before Anti-VEGF After Anti-VEGF

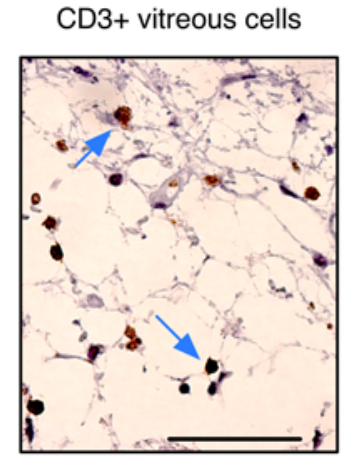

G Differentially-expressed pathways
H

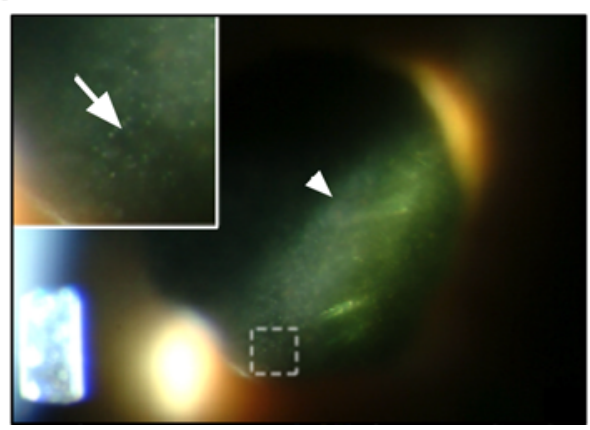

I

Anterior Chamber Cells

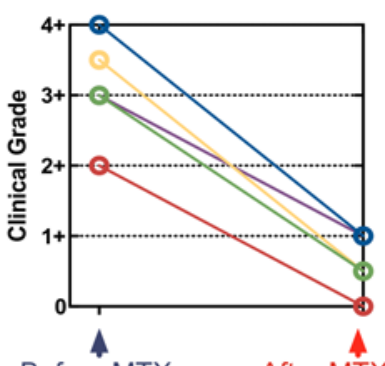

Before MTX

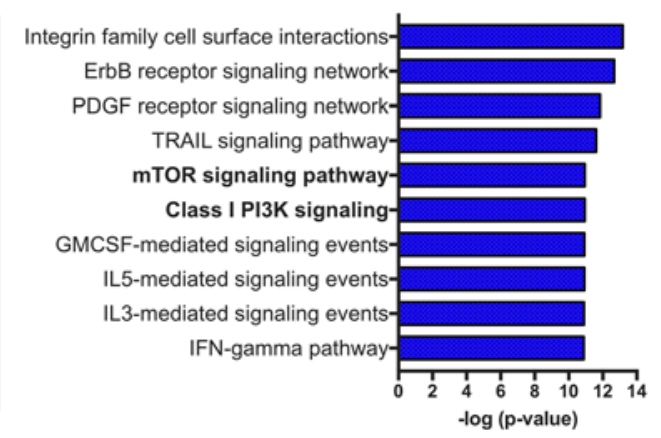

Vascular Leakage (Flare)

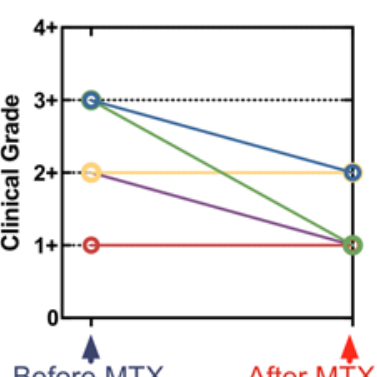

Before MTX After MTX

Figure 2. Personalized proteomes guide rational therapeutic repurposing for autoinflammatory disease. (A) VEGF overexpression increases as neovascular inflammatory vitreoretinopathy (NIV) severity progresses, implicating anti-VEGF injectable therapeutics as potential NIV therapy. In contrast, TNF- $\alpha$ was never overexpressed in NIV eyes, explaining why injecting infliximab always failed. (B) Fundoscopic examination of a patient with stage III NIV with marked vitreous hemorrhage (VH) whose vision was reduced to "count fingers" (CF). This patient received an injection of bevacizumab (anti-VEGF). Fundoscopic examination, after injection of the therapeutic anti-VEGF antibody vitreous hemorrhage (arrow head) is resolved as vision improved to $20 / 70$ (C) at 2 weeks and 20/50 (D) at 4 weeks. As the vitreous hemorrhage resolves (arrow head), the optic nerve head (open arrow) becomes visible. (E) Vitreous hemorrhage grade resolved after bevacizumab injection in 7 NIV eyes. (F) CD3-positive T cells (arrows) found in the vitreous of donor NIV eyes. Scale bar: $50 \mu \mathrm{m}$. (G) Pathway analysis revealed changes in cytokine expression in all stages of NIV. Graph of results shows the 10 pathways most affected. Pathways are organized by the $\log (P$ value $)$ obtained from the right-tailed Fisher exact test. The class I PI3K and mTOR signaling pathways are known to be linked to T cell development. (H) Slit-lamp examination of a NIV patient with posterior uveitis showing vascular leakage of protein (flare; arrow head) and anterior chamber cells (inset; open arrow). (I) Postoperative, intravitreal injections of methotrexate (MTX) reduced anterior chamber cell grade, but did not reduce tissue damage and vascular leakage of protein into the vitreous.

they do develop retinal neovascularization similar to some uveitis patients (5). We considered repurposing bevacizumab for treatment of NIV.

For NIV patients, vitreous hemorrhages were removed through vitrectomy surgery, but this surgery triggered an exaggerated postoperative inflammatory response that accelerated inflammation and fibrosis. 
Because of the elevated VEGF levels detected in our proteomics analysis, we tested intravitreal bevacizumab injection in several NIV patients. Figure 2B shows an eye filled with blood in one patient whose sight was reduced to "count fingers" (CF), but a single injection of bevacizumab improved their vitreous hemorrhage grade and visual acuity to 20/70 two weeks later (Figure 2C), and to their baseline of 20/50 four weeks later (Figure 2D). We have used anti-VEGF therapy in 7 eyes to resolve such hemorrhages without surgery, and in each case the vitreous hemorrhage grade resolved and their vision returned to baseline (Figure 2E and Supplemental Table 5). Thus, repurposing a cancer and macular degeneration drug was a safer, less invasive approach, and less expensive than surgery. Bevacizumab has since been repurposed to treat neovascularization secondary to retinal ischemia (i.e., diabetic retinopathy) — an anatomically and pathologically distinct phenotype not present in NIV or uveitis patients (6).

Inflammatory cell inhibition. Although the bevacizumab injections stopped neovascularization in NIV patients, other problems persisted, including cellular inflammation and vascular leakage of protein into the eye, which is visible as flare (in which proteins in the vitreous and anterior chamber can scatter a beam of light). The cytokines uniquely upregulated in NIV (compared with controls) were queried using databases for pathway analysis to assess proteins that were differentially expressed in NIV and not found in control, uninflamed, human eyes (Figure $2 \mathrm{G}$ ). We then reviewed databases to identify small molecules and biologics to target these upregulated cytokine pathways.

Of the highly represented pathways, the mTOR and class I PI3K signaling pathways were identified ( $P$ $<0.005$; Figure 2G). These pathways are known to be critical in determining the fate of developing $\mathrm{T}$ cells. In fact, there were CD3-positive T cells in the vitreous fluid of donor NIV eyes (Figure 2F) (7). In the past, vitreous inflammation was treated with intraocular corticosteroid injections, but this puts NIV patients at risk for glaucoma (8). We considered sirolimus because of the presence of mTOR effectors in NIV vitreous. However, oral sirolimus was reported to have limited efficacy in treating uveitis due to its undesirable side effects (9), and there was no intravitreal formulation available at the time of the study (10). In seeking alternative drug repositioning options, we noted that methotrexate could target $\mathrm{T}$ cells in both healthy and rheumatoid arthritis patients, could effectively suppress ocular inflammation, and was safe for intravitreal injection (11-18). Although our previous use of oral methotrexate was ineffective, the proteomic data and ability to dramatically increase local drug concentration by injection influenced us to reconsider methotrexate. Therefore, we repurposed methotrexate to alleviate NIV-specific T cell inflammation. After treating 5 eyes with stage II and stage III NIV using intravitreal injections of methotrexate $(400 \mu \mathrm{g} / 0.1 \mathrm{ml})$, the number of cells in the anterior chamber dropped dramatically (Figure 2, H and I, and Supplemental Table 6). Repurposing methotrexate was so uniformly successful in the clinic that we routinely use intravitreal methotrexate perioperatively, to mitigate the exuberant postsurgical inflammation in NIV patients. NIV patients can now tolerate eye surgery without a damaging immune response.

Interestingly, despite improvements with intravitreal methotrexate, anterior chamber flare persisted (Figure 2I). This suggested methotrexate had specifically prevented proliferation of inflammatory cells within the eye, but did not resolve the disruption in the blood-ocular barrier that allowed protein to leak into the vitreous.

Corticosteroid implant. In previous treatment, oral prednisone showed some benefit, but was not tolerated due to systemic side effects (19) such as weight gain and osteoporosis. Since our personalized proteome showed many of the cytokines upregulated by NIV should be quelled by corticosteroid therapy, we instead chose to deliver corticosteroid nonsystemically, by surgically implanting fluocinolone acetonide (Retisert) implants into 3 eyes that represented stage II and III disease. This implant delivers continuous steroid for over 2 years, reducing the need for frequent injections. Implanted patients showed reversal of several clinical features of stage II and III NIV: retinal neovascularization was halted and the number of intraocular leukocytes dropped. Proteomic analysis of vitreous biopsies taken before and after the steroid implant (Supplemental Figure 2, A-C, and Supplemental Table 7) indicated that intraocular VEGF, VEGFR3, PDGFR $\beta$, FGF-4, and FGF-7 levels fell, which likely helped resolve the retinal neovascularization. In addition, 26 other cytokines linked to leukocyte infiltration were also reduced to control levels (Supplemental Figure 2, A-C, and Supplemental Table 8).

IL-6 inhibition. Despite these clinical improvements after Retisert implantation, severe intraocular fibrosis persisted (20), suggesting that some cytokines were insensitive to corticosteroid therapy. Therefore, we sought a repositioning strategy to target fibrosis. The elevated IL-6 levels that remained after Retisert implantation (and in stage IV disease) were an attractive target (Supplemental Figure 2C and 
A
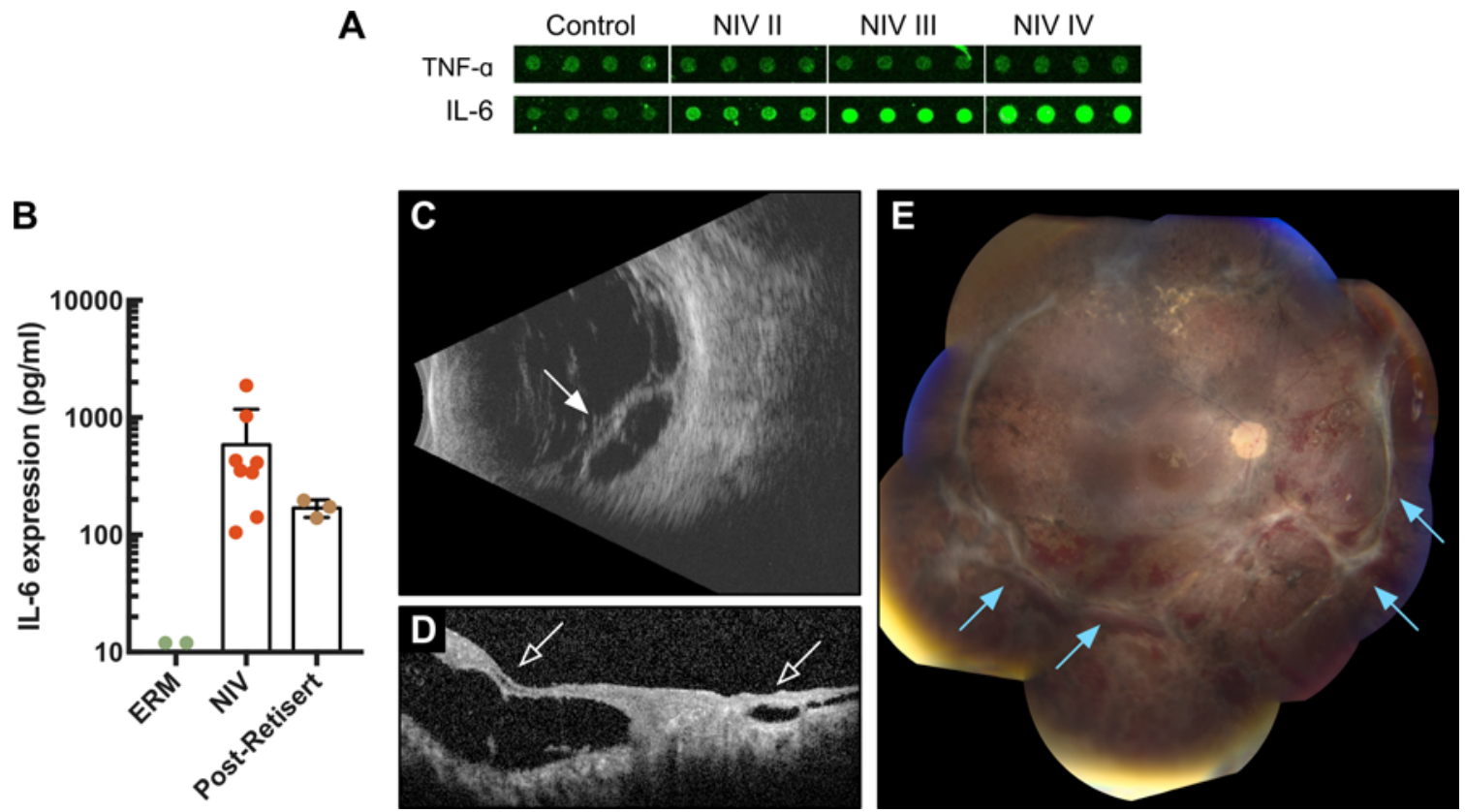

Figure 3. Targeting IL-6 levels blocks fibrosis and allows retinal reattachment. (A) IL-6 expression in neovascular inflammatory vitreoretinopathy (NIV) showing increased expression throughout disease progression. (B) Vitreous IL-6 levels were measured using ELISA in control (epiretinal membrane $[\operatorname{ERM}])(n=4)$, NIV $(n=8)$, and post-Retisert eyes $(n=3)$. Results (in pg/ml) are displayed as mean \pm SD. (C) Posterior B-scan of a patient with advanced NIV and recurrent retinal detachment with fibrosis (arrow). (D) Spectral domain optical coherence tomography (SD-OCT) imaging showing tractional retinal detachment (open arrow). (E) Patient underwent retinal reattachment surgery with intravitreal injection of tocilizumab (anti-IL-6). Postoperative posterior B-scan reveals successful retinal reattachment 6 months after surgery. Fundoscopic examination of advanced NIV patient following personalized therapy.

Figure 3, A and B), since IL-6 is linked to many fibrotic diseases (21-28), including ocular fibrosis associated with proliferative diabetic retinopathy (29) and proliferative vitreoretinopathy (30). Tocilizumab, which targets IL-6 (anti-IL-6), is used to treat severe cases of rheumatoid arthritis, systemic juvenile idiopathic arthritis, and is currently in trial for the treatment of systemic sclerosis $(28,31)$. So, for a special case of stage IV NIV, we considered repurposing tocilizumab.

Our repositioning strategies came together in 1 NIV patient, who was monocular due to chronic intraocular fibrosis and retinal detachment in her left eye (stage IV). In what had been her functional right eye, she recently developed a severe fibrotic, neovascular retinal detachment (Figure 3C). Repair of fibrotic retinal detachment requires a long, delicate surgery to manually remove the scar tissue; however, in NIV eyes, scar tissue regrows within weeks and causes tractional and recurrent retinal detachment and poor visual outcomes. The fibrosis is so aggressive that, for the most part, we had stopped retinal reattachment surgery on NIV eyes. To save the sight in this patient's only functioning eye, an alternative therapy was needed. The patient underwent a retinal detachment repair surgery combined with our proteomic-driven approach - Retisert, anti-VEGF antibody, and intravitreal methotrexate at the time of surgery. Although neovascularization and cellular inflammation were suppressed, retinal fibrosis recurred within weeks like other NIV eyes and her retina re-detached (Figure 3D). We knew that IL-6 expression was not controlled with our previous therapies; therefore, after a second surgery to reattach her retina, she was given monthly intravenous infusions of tocilizumab (anti-IL-6) over 6 months. Remarkably, blocking IL-6 with tocilizumab allowed her retina to remain attached, a first for retinal reattachment surgery in a NIV patient (Figure 3E). Although this is a single case, these results in a very aggressive form of NIV strongly suggest that IL-6 is linked to retinal fibrosis and repurposing anti-IL-6 therapy can control intraocular fibrosis. At the time of our therapeutic repurposing, tocilizumab was not available for off-label use. Since then, tocilizumab has been shown to be effective in the treatment of some refractory uveitis cases and other ocular inflammatory diseases. This, combined with elevated IL-6 levels at each stage of NIV, make tocilizumab an attractive first-line therapy in future NIV cases (Figure 1B and Figure 3A). 


\section{Discussion}

Using proteomics in real time, we detected dynamic changes in inflammatory processes and identified targets that were treatable, allowing us to reposition clinical therapies immediately. Thus, cytokine profiles of NIV eyes guided our drug repositioning strategies for a previously untreatable population and leveraged the enormous effort already invested in approved, available drugs. Since, regardless of the tissue affected (e.g., kidney, liver, joints), inflammatory diseases can be characterized by cytokine signatures, our proof of principle with NIV offers an excellent model for developing drug repositioning strategies for other chronic inflammatory diseases. We believe proteomic analysis of biopsy fluid might well, in the future, allow physicians to take a snapshot of other inflammatory disease processes, in real time, and similarly guide optimal repositioning of approved drugs.

Drug repositioning is a promising innovation since repurposed drugs are already FDA-approved, have proven bioavailabilities, and well-characterized side-effect profiles. The approach can provide a safer alternative to the development of new compounds (2), but the question remains, how can clinicians select the appropriate drug for repurposing? Personalized analysis of liquid biopsies transformed our treatment approach for NIV patients. Before our proteomic study, treatment was trial and error (and largely ineffective). Proteomic analysis of liquid biopsies from patients of various NIV stages gave us insight into the disease states at the molecular level. Knowing which molecular pathways were abnormally activated, we searched databases of available drugs that could target these pathways, and found we had ready access to the drugs to treat NIV. What we had needed was not new drugs, but a way to determine which available drugs were likely to have an effect. We now treat vitreous hemorrhages with bevacizumab, instead of surgery. Intravitreal methotrexate is used to control the infiltration of inflammatory cells and the exaggerated inflammatory response to surgery. Retisert implants slow the progression of the disease, but not the fibrotic stage. Now, we are also considering IL-6 therapy to prevent retinal fibrosis (Supplemental Figure 3). Importantly, based on previous failures and the lack of TNF- $\alpha$ in NIV biopsy samples, we have completely stopped giving anti-TNF- $\alpha$ therapy. We have also stopped treating the fibrotic stage (IV) with corticosteroids. One aspect that we have yet to effectively treat is the early photoreceptor synaptic signaling defect (loss of b-wave on electroretinogram) that heralds the progressive retinal degeneration. Addressing the neurodegenerative aspect of NIV will require further molecular characterization of cell death biomarkers rather than cytokine biomarkers in NIV eyes. Our investigations of animal models suggest that retinal biomarkers may also be detected in liquid vitreous biopsies (32-34).

Our experience with NIV implies the same approach might extend to other inflammatory diseases in which liquid biopsies from affected sites might pinpoint pathological changes in real time. NIV patients have shared a genetic origin with clear phenotypic stages. In this case, the clinical staging is now correlated with a molecular phenotype, which allows for immediate treatment of new NIV patients without further molecular profiling. For other inflammatory diseases with limited or complex phenotypic characterization, individual cytokine levels could be directly measured to guide treatment. Thus, without understanding the molecular origins of the disease state, as implied by genetic testing or even a definitive diagnosis, proteomics might guide treatment and allow the use of available, approved treatments that otherwise might not be offered. This approach clearly could be extended to more comprehensive proteomic analysis, and applied to other tissue biopsy samples, to describe disease states precisely and personalize treatment options.

In the future, proteomic profiling of liquid biopsies should yield the personalized benefits that had been expected of genetic testing. Genetic testing may have limits for drug repositioning if it only estimates the risk patients have for acquiring a disease. In contrast, proteomic profiling of liquid biopsies identifies realtime effectors of expressed genes, precisely reflecting the health or disease of surrounding tissues, regardless of a patient's genotype, and thereby provide a snapshot of a disease state in real time. This type of analysis can be used to guide diagnosis and treatment, and assess follow-up.

Our study was a retrospective analysis in a small sample size, but our results are strong because all our subjects all carry the identical rare genetic variant. Similar assessments of unrelated patients would require larger study populations, but our success with this small group suggests that those types of studies are worthwhile. Indeed, many diseases that we currently deem untreatable might be well controlled with drugs that are available and in use. We expect that proteomic analysis of fluid biopsies is likely to become routine, and we are likely to find we already have the power to treat diseases that are thought to have no available drugs. 


\section{Methods}

Vitreous sample collection. Pars plana vitrectomy was performed using a single-step transconjunctival 23-gauge trocar cannular system (Alcon Laboratories Inc.), and an undiluted $0.5-\mathrm{ml}$ sample of the vitreous was manually aspirated into a 3-ml syringe. Vitreous samples were immediately centrifuged in the operating room at $15,000 \mathrm{~g}$ for 5 minutes at room temperature to remove impurities and then finally stored at $-80^{\circ} \mathrm{C}$, as previously described (35).

Cytokine array. Vitreous cytokine-signaling proteins were measured using the Human Cytokine Quantibody Array 4000 (RayBio) per the manufacturer's protocol. This array concurrently detected and processed 200 human cytokines. First, the array chips were incubated with sample diluents for 30 minutes at room temperature to act as a block. Vitreous sample volumes under $500 \mu 1$ were diluted with buffer to reach a required sample volume (Supplemental Table 2). Vitreous (100 $\mu 1 ; 4$ technical replicates per sample) was then added to the wells of the array and incubated overnight at $4{ }^{\circ} \mathrm{C}$. A standard cytokine dilution was added to the wells of the array to determine protein concentrations. For signal detection, $80 \mu 1$ of $\mathrm{Cy} 3$ streptavidin was added to each well, rinsed, and visualized by laser scanner. The RayBio Analysis Tool was used for protein classification. Final cytokine concentrations (in $\mathrm{pg} / \mathrm{ml}$ ) were corrected for sample dilution (Supplemental Table 2). Additional quality-control data for the array are provided in the online supplement (Supplemental Table 3 and Supplemental Figure 1).

Intervention/surgery. Intravitreal injections were performed in the same manner in all patients. After anesthetization with $0.1-0.3 \mathrm{ml}$ of $1 \%$ lidocaine into the subconjunctival space, a lid speculum was placed and $5 \%$ povidone-iodine placed onto the conjunctival surface. Calipers were used to mark $3.5 \mathrm{~mm}$ from the limbus and either methotrexate $(400 \mu \mathrm{g} / 0.1 \mathrm{ml})$ or bevacizumab $(1.25 \mu \mathrm{g} / 0.05 \mathrm{ml})$ was injected into the center of the vitreous cavity through a 30-gauge needle. A cotton-tip applicator was held at the site of the injection as the needle was withdrawn. All patients were instructed to instill one drop of a fourthgeneration fluoroquinolone 4 times a day into the affected eye(s) for 4 days following the injection (4). Clinical outcomes for these patients are detailed in the online supplement (Supplemental Tables 5 and 6). Fluocinolone acetonide (Retisert) devices were implanted as previously described (20).

Immunohistochemistry. Postmortem NIV eyes (University of Iowa, Department of Pathology archived tissue collection) were received in formalin and postfixed in Pen-fix (Thermo Fisher Scientific). After the eye was opened by pupil-optic nerve section, it was decalcified. Immunohistochemical staining was performed as follows: Slides were stained on the DAKO Autostainer+, using heat pretreatment with a pressure cooker. The anti-CD3 (DAKO, A0452) diluted to 1:200 was incubated for 30 minutes. A dual endogenous enzyme block (DAKO, S2003) was used for 5 minutes. Detection was for 30 minutes and DAB+ (DAKO, K3467) was used for 5 minutes. DAKO Envision+ Dual-Link labeled polymer (K4061) was used for detection.

Statistics. Following cytokine concentration detection, data were sorted on Microsoft Excel spreadsheets based on concentration hierarchy. Data were sorted into 2 sections: control patients with macular holes and epiretinal membranes and NIV patients. Cytokines present in any of the control or patients with NIV at a level of $500 \mathrm{pg} / \mathrm{ml}$ or higher were selected for further evaluation. This cutoff was chosen because it is higher than the sensitivity of the assay $(500 \mathrm{pg} / \mathrm{ml})$, but a low threshold to catch all significantly expressed proteins while removing the underexpressed or absently expressed proteins from the list. Results were also saved in Excel as .txt format and were uploaded into the Partek Genomics Suite 6.5 software package. The data for controls and NIV patients were normalized to log base 2 , and compared using 1-way ANOVA. All proteins with nonsignificant $(P \geq 0.05)$ changes were eliminated from the table. The significant values were mapped using the 'cluster based on significant genes' visualization function with the standardization option. Pathway representation of upregulated cytokines was performed using Pathway Commons and WikiPathways analysis in WebGestalt (36).

Study approval. The study was approved by the University of Iowa's Institutional Review Board, and adhered to the tenets set forth in the Declaration of Helsinki. Data were collected and analyzed from August 2009 to April 2017. Our proteomics analysis was used to collect information on the NIV vitreous proteome at different stages of disease in 1 set of NIV patients (Supplemental Table 1). These patients that provided research biopsies were not treated based on their own array data. Therapies were selected in a different set of NIV patients based on their stage of disease after failure of conventional therapies. Patients were consented regarding the off-label use of therapies and the known benefits, risks, and past uses of therapies for ocular disease. Subjects underwent eye exams that included slit-lamp examination, dilated retinal biomicroscopy, and indirect ophthalmoscopy. Written informed content was provided for pictures appearing in the manuscript. 
Standard-of-care testing was performed in all cases to determine the etiology of the uveitis. Infectious etiologies tested for were toxoplasmosis IgG and IgM, syphilis IgG, Lyme disease and quantiferon gold. Autoimmune testing included angiotensin-converting enzyme, chest x-ray, anti-nuclear antibody, and rheumatoid factor. In cases of suspected viral infection, PCR of aqueous fluid was performed for varicella zoster, herpes simplex, and cytomegalovirus.

\section{Author contributions}

VBM had full access to all the data in the study and takes responsibility for the integrity of the data and the accuracy of the data analysis. VBM, SHT, and AGB conceived and designed the study. GV and VBM acquired data. GV, DC, SHT, AGB, and VBM analyzed and interpreted data. GV, DC, SHT, AGB, and VBM drafted the manuscript. SHT, AGB, and VBM critically revised the manuscript for important intellectual content. GV and VBM performed statistical analyses. VBM obtained funding, provided administrative, technical, and material support, and supervised the study.

\section{Acknowledgments}

We wish to thank Polly Ferguson and John Colgan for critical review of the manuscript.

Funding/support: VBM and AGB are supported by NIH grants (R01EY026682, R01EY024665, R01EY025225, R01EY024698, R21AG050437, and P30EY026877), The Doris Duke Charitable Foundation grant 2013103, and Research to Prevent Blindness (RPB), New York, NY. GV is supported by NIH grants (F30EYE27986 and T32GM007337). The Barbara \& Donald Jonas Laboratory of Regenerative Medicine and Bernard \& Shirlee Brown Glaucoma Laboratory are supported by NIH grants (5P30EY019007, R01EY018213, R01EY024698, and R21AG050437), the National Cancer Institute Core (5P30CA013696), the RPB Physician-Scientist Award, and unrestricted funds from the RPB. SHT is a member of the RD-CURE Consortium and is supported by the Tistou and Charlotte Kerstan Foundation, the Schneeweiss Stem Cell Fund, New York State (C029572), the Joel Hoffman Fund, the Professor Gertrude Rothschild Stem Cell Foundation, and the Gebroe Family Foundation.

Address correspondence to: Vinit B. Mahajan, Byers Eye Institute, Department of Ophthalmology, Stanford University, 1651 Page Mill Road, Palo Alto, California, 94304 USA. Phone: 650.723.6995; Email: vinit.mahajan@stanford.edu.

1. Ashburn TT, Thor KB. Drug repositioning: identifying and developing new uses for existing drugs. Nat Rev Drug Discov. 2004;3(8):673-683.

2. Novac N. Challenges and opportunities of drug repositioning. Trends Pharmacol Sci. 2013;34(5):267-272.

3. Wert KJ, et al. CAPN5 mutation in hereditary uveitis: the R243L mutation increases calpain catalytic activity and triggers intraocular inflammation in a mouse model. Hum Mol Genet. 2015;24(16):4584-4598.

4. Mahajan VB, et al. Bilateral intravitreal injection of antivascular endothelial growth factor therapy. Retina (Philadelphia, Pa). 2011;31(1):31-35.

5. Patel AK, et al. Risk of retinal neovascularization in cases of uveitis. Ophthalmology. 2016;123(3):646-654.

6. Ferrara N, Adamis AP. Ten years of anti-vascular endothelial growth factor therapy. Nat Rev Drug Discov. 2016;15(6):385-403

7. Mahajan VB, et al. T-cell infiltration in autosomal dominant neovascular inflammatory vitreoretinopathy. Mol Vis. 2010;16:1034-1040

8. Jones R, Rhee DJ. Corticosteroid-induced ocular hypertension and glaucoma: a brief review and update of the literature. Curr Opin Ophthalmol. 2006;17(2):163-167.

9. Phillips BN, Wroblewski KJ. A retrospective review of oral low-dose sirolimus (rapamycin) for the treatment of active uveitis. J Ophthalmic Inflamm Infect. 2010;1(1):29-34.

10. Nguyen QD, et al. Intravitreal sirolimus for noninfectious uveitis: a phase III Sirolimus Study Assessing Double-masKed Uveitis TReAtment (SAKURA). Ophthalmology. 2016;123(11):2413-2423.

11. Cutolo M, Sulli A, Pizzorni C, Seriolo B, Straub RH. Anti-inflammatory mechanisms of methotrexate in rheumatoid arthritis Ann Rheum Dis. 2001;60(8):729-735.

12. Gerards AH, de Lathouder S, de Groot ER, Dijkmans BA, Aarden LA. Inhibition of cytokine production by methotrexate. Studies in healthy volunteers and patients with rheumatoid arthritis. Rheumatology (Oxford). 2003;42(10):1189-1196.

13. Johnston A, Gudjonsson JE, Sigmundsdottir H, Ludviksson BR, Valdimarsson H. The anti-inflammatory action of methotrexate is not mediated by lymphocyte apoptosis, but by the suppression of activation and adhesion molecules. Clin Immunol. 2005;114(2):154-163.

14. Hardwig PW, Pulido JS, Erie JC, Baratz KH, Buettner H. Intraocular methotrexate in ocular diseases other than primary central nervous system lymphoma. Am J Ophthalmol. 2006;142(5):883-885. 
15. Taylor SR, Habot-Wilner Z, Pacheco P, Lightman SL. Intraocular methotrexate in the treatment of uveitis and uveitic cystoid macular edema. Ophthalmology. 2009;116(4):797-801.

16. Genestier L, Paillot R, Fournel S, Ferraro C, Miossec P, Revillard JP. Immunosuppressive properties of methotrexate: apoptosis and clonal deletion of activated peripheral T cells. J Clin Invest. 1998;102(2):322-328.

17. Smith JR, et al. Role of intravitreal methotrexate in the management of primary central nervous system lymphoma with ocular involvement. Ophthalmology. 2002;109(9):1709-1716.

18. Teachey DT, et al. mTOR inhibitors are synergistic with methotrexate: an effective combination to treat acute lymphoblastic leukemia. Blood. 2008;112(5):2020-2023.

19. Schäcke H, Döcke WD, Asadullah K. Mechanisms involved in the side effects of glucocorticoids. Pharmacol Ther. 2002;96(1):23-43.

20. Tlucek PS, Folk JC, Orien JA, Stone EM, Mahajan VB. Inhibition of neovascularization but not fibrosis with the fluocinolone acetonide implant in autosomal dominant neovascular inflammatory vitreoretinopathy. Arch Ophthalmol. 2012;130(11):1395-1401.

21. Shahar I, et al. Effect of IL-6 on alveolar fibroblast proliferation in interstitial lung diseases. Clin Immunol Immunopathol. 1996;79(3):244-251.

22. Lin ZQ, Kondo T, Ishida Y, Takayasu T, Mukaida N. Essential involvement of IL-6 in the skin wound-healing process as evidenced by delayed wound healing in IL-6-deficient mice. J Leukoc Biol. 2003;73(6):713-721.

23. Canataroglu H, Varinli I, Ozcan AA, Canataroglu A, Doran F, Varinli S. Interleukin (IL)-6, interleukin (IL)-8 levels and cellular composition of the vitreous humor in proliferative diabetic retinopathy, proliferative vitreoretinopathy, and traumatic proliferative vitreoretinopathy. Ocul Immunol Inflamm. 2005;13(5):375-381.

24. Barnes TC, Anderson ME, Moots RJ. The many faces of interleukin-6: the role of IL-6 in inflammation, vasculopathy, and fibrosis in systemic sclerosis. Int J Rheumatol. 2011;2011:721608.

25. Cui W, Zhang H, Liu ZL. Interleukin-6 receptor blockade suppresses subretinal fibrosis in a mouse model. Int J Ophthalmol. 2014;7(2):194-197.

26. Fielding CA, et al. Interleukin-6 signaling drives fibrosis in unresolved inflammation. Immunity. 2014;40(1):40-50

27. Le TT, et al. Blockade of IL-6 trans signaling attenuates pulmonary fibrosis. J Immunol. 2014;193(7):3755-3768.

28. Khanna D, et al. Safety and efficacy of subcutaneous tocilizumab in adults with systemic sclerosis (faSScinate): a phase 2, randomised, controlled trial. Lancet. 2016;387(10038):2630-2640.

29. Mocan MC, Kadayifcilar S, Eldem B. Elevated intravitreal interleukin-6 levels in patients with proliferative diabetic retinopathy. Can J Ophthalmol. 2006;41(6):747-752.

30. Mahajan VB, Roybal CN, Velez G. Personalized proteomics for proliferative vitreoretinopathy. Abstract presented at: American Eye Study Club 2017 Annual Meeting; July 30-August 4, 2017; Dana Point, California.

31. Kihara M, et al. Use and effectiveness of tocilizumab among patients with rheumatoid arthritis: an observational study from the British Society for Rheumatology Biologics Register for rheumatoid arthritis. Clin Rheumatol. 2017;36(2):241-250

32. Skeie JM, Roybal CN, Mahajan VB. Proteomic insight into the molecular function of the vitreous. PLoS ONE. 2015;10(5):e0127567.

33. Skeie JM, Mahajan VB. Proteomic interactions in the mouse vitreous-retina complex. PLoS ONE. 2013;8(11):e82140.

34. Mahajan VB, Skeie JM. Translational vitreous proteomics. Proteomics Clin Appl. 2014;8(3-4):204-208.

35. Velez G, Roybal CN, Colgan D, Tsang SH, Bassuk AG, Mahajan VB. Precision medicine: personalized proteomics for the diagnosis and treatment of idiopathic inflammatory disease. JAMA Ophthalmol. 2016;134(4):444-448.

36. Zhang B, Kirov S, Snoddy J. WebGestalt: an integrated system for exploring gene sets in various biological contexts. Nucleic Acids Res. 2005;33(Web Server issue):W741-W748 